\title{
Exciton-polariton emission from organic semiconductor optical waveguides
}

\author{
Tal Ellenbogen* ${ }^{*}$ and Kenneth B. Crozien \\ Harvard School of Engineering and Applied Sciences, \\ 33 Oxford Street, Cambridge, Massachusetts 02138, USA
}

\begin{abstract}
We photo-excite slab polymer waveguides doped with J-aggregating dye molecules and measure the leaky emission from strongly coupled waveguide exciton polariton modes at room temperature. We show that the momentum of the waveguide exciton polaritons can be controlled by modifying the thickness of the excitonic waveguide. Non-resonantly pumped excitons in the slab excitonic waveguide decay into transverse electric and transverse magnetic strongly coupled exciton waveguide modes with radial symmetry. These leak to cones of light with radial and azimuthal polarizations.
\end{abstract}

The emission of light by recombination of electronhole pairs (excitons) in excitonic materials, e.g. semiconductors, quantum wells, dye molecules, is used for a wide range of applications, including lasers, light emitting diodes [1, and fluorescent tags in biology [2]. The majority of the applications based on photoemission from excitons operate in the weak coupling regime, where the exciton is annihilated and as a consequence a photon is emitted either by spontaneous emission or by stimulated emission. The exciton and emitted photon can therefore be treated as two separate physical entities. On the other hand, in the strong coupling regime, the exciton and photon mode can exchange energy at a rate faster than the decay rate of the exciton and the escape rate of the photon, and the two particles create a hybrid state known as exciton-polariton (EP) [3, 4]. The EP can be considered as a quasi particle with mixed properties of light and matter. The masses of cavity EPs are $10^{-4}-10^{-5}$ times those of typical semiconductor excitons [5]. In comparison to bare photons which cannot scatter each other, EPs can scatter each other thanks to their excitonic part, leading to strong population-dependent nonlinearities [6, 7] and interesting physical phenomena, e.g. Bose-Einstein condensation (BEC) which results in macroscopic coherence of the condensate and superfluidity [8] 10 . Furthermore, these hybrid light matter states have been shown to allow new device applications such as the generation of low threshold coherent emission at room temperature without the need for population inversion [6-12] and low threshold all-optical switches based on exciton-polariton scattering 13.

To date, most EP studies and devices have been demonstrated using optical cavities 3 14]. In optical cavities, strong confinement of the optical modes makes reaching the strong coupling regime possible, and the dispersion relation of the cavity EP exhibits a potential well, allowing BEC of the EPs into the lowest energy state. However, this configuration is somewhat limited since the EP modes of the system are localized and cannot propagate, and cannot be probed directly. In addition these devices, being based on cavities, are usually rigid and are sensitive to deformations [10]. This motivates the investigation of configurations other than cavities that allow strong coupling of electromagnetic modes with excitons.

Recently it was shown that strong coupling can occur between excitons and surface plasmon polaritons with large room temperature Rabi-splitting values [15-17]. Another optical configuration that strongly confines photons, and therefore can be considered for strong coupling with excitons, is an optical waveguide. This configuration is both common and highly suitable for applications, but has received only modest attention in the context of strongly coupled modes [18 21]. Waveguide modes are advantageous compared to cavity modes since they can propagate, can be studied and directly probed at the near field through their evanescent tails and the devices can be deformed with little change to the modes of the system, in the same way that optical fibers can be bent with little effect. Studying strong coupling in this configuration could potentially provide new insights into EPs and could permit new applications and devices.

We have recently shown that the eigenmodes of a thin polymer waveguide doped with J-aggregate excitons are strongly coupled waveguide-exciton-polariton (WGEP) modes [22]. This was validated by numerical studies and experimental reflectivity measurements of the dispersion relation. The strong coupling phenomenon was seen to manifest itself with large splitting in the curve of the dispersion relation of both the fundamental transverse electric (TE) and transverse magnetic (TM) waveguide modes of the system. We also showed that the strength of the coupling between the excitons and the TE waveguide modes is similar to that of excitons and surface plasmon polariton modes in the same system, where the waveguide mode benefits from lower losses.

Here we study the emission properties of J-aggregating dye molecules embedded in polymer optical waveguides. We show that there is strong leaky emission from WGEP modes to angles corresponding to the phase matching conditions between the fundamental WGEP modes and leaky photons. We experimentally confirm that, much like cavity EPs, the coupling strength between the optical waveguide modes and excitons is proportional to the square root of the absorbance in the J-aggregates. Therefore, the large oscillator strengths of the J-aggregate excitons make it possible to observe this phenomenon at 


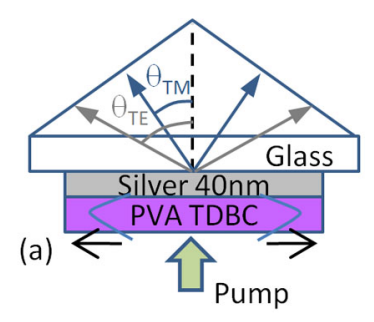

(b)

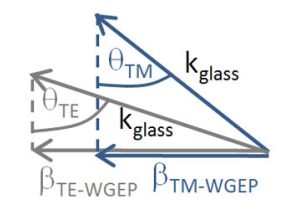

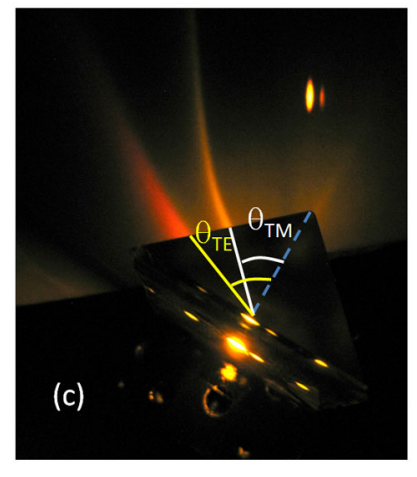

Figure 1: (color online). (a) Schematic representation of the sample mounted on a prism. (b) Momentum conservation diagram. (c) Photograph of leaky emission from the sample projected on a white screen.

room temperature. We show that, due to the extended photonic part of WGEP modes, we can control their momentum by appropriate choice of the thickness of the excitonic polymer waveguide. We further show that nonresonantly pumped excitons decay into WGEPs with radial symmetry, which leak to cones of light in free space that have radial and azimuthal polarizations.

To fabricate the EP slab waveguide we deposit a thin silver film (40 nm thick) on glass by thermal evaporation, followed by spin coating an aqueous solution of $5 \%$ polyvinyl alcohol (PVA) mixed in a 3:1 ratio with a 6.6 $\mathrm{mM}$ solution of J-aggregating cyanine dye. The cyanine dye used is the 5,6-dichloro-2-[3-[5,6-dichloro-1-ethyl3-(3-sulfopropyl)-2(3H)-benzimidazolidene]-1-propenyl]1-ethyl-3-(3-sulfopropyl) benzimidazolium hydroxide, inner salt, sodium salt, NK2203, Hayashibara (TDBC), which is known to form linear chains of J-aggregating molecules at low concentrations $(>0.05 \mathrm{mM})$ with large oscillator strengths [23]. The thickness of the spin coated polymer film is measured by a profilometer to be $\backsim 275$ nm (Veeco Dektak 6M). The doped polymer film acts as the optical waveguide in our system. The thin metal film separates the polymer waveguide and the glass substrate, enabling optical confinement in the polymer slab, which would otherwise be difficult due to their very similar indices of refraction $\left(\lambda=590 \mathrm{~nm}: n_{B K 7}=1.52\right.$, $\left.n_{P V A}=1.49-1.55[24]\right)$. The metal film is kept thin enough to allow optical coupling to the waveguide mode from the substrate side.

A schematic representation of the sample mounted on a glass prism is shown in Fig. 1(a). Index matching oil is used in between the sample and prism. The excitons are non-resonantly pumped from the waveguide side and relax into the eigenmodes of the system which are WGEP modes. These hybrid modes couple out to photons through the prism in accordance with the mo- mentum conservation condition at the boundary:

$$
k_{\text {glass }} \sin (\theta)=\beta_{W G E P}
$$

where $k_{\text {glass }}=2 \pi n_{\text {glass }} / \lambda$ is the momentum of the leaky photons in the glass, $n_{\text {glass }}$ is the refractive index, $\lambda$ is the free space wavelength, $\beta_{W G E P}$ is the momentum of the WGEP and $\theta$ is leakage angle. Fig 1(b) depicts the momentum conservation conditions for the TE and the TM modes of the system. TE and TM WGEP modes carry different momenta and thereby couple out of the sample to different angles through the prism. Fig. 1(c) shows a photograph of the leaky emission through the prism projected on a screen when the sample is pumped by a continuous wave $(\mathrm{CW})$ laser diode with a wavelength of $532 \mathrm{~nm}$. It should be noted that emission also occurs on the right side of the prism, but cannot be seen in Fig. 1(c) as the placement of the screen only captures the emission from the left side. A $550 \mathrm{~nm}$ long pass filter is used at the camera to block scattered light from the excitation source. Emission into two distinct angles is clearly observed. The two beams are linearly polarized where the emission into $\theta_{T E}$ is polarized parallel to the silver film (s polarized) and the emission into $\theta_{T M}$ is polarized in the plane of the illustration shown in Fig. 1(a) (p polarized).

To perform far field spectral analysis of the leaky emission from the sample, the sample is non-resonantly pumped with a $488 \mathrm{~nm}$ CW laser from the polymer film side (see Fig. 1(a)). The leaky emission is focused by an objective lens into an optical fiber, and input to a spectrometer with electrically cooled CCD detector (Synapse, Horiba Scientific). The collection arm is mounted on a rotation stage, enabling the angle of collection, $\theta$, to be changed. Fig. 2 (a) shows the leaky emission spectrum collected at $\theta_{T E}=61.2^{\circ}$. Peaks can be seen at $\lambda=$ $536 \mathrm{~nm}, 637 \mathrm{~nm}$ and $600 \mathrm{~nm}$, corresponding to leaky emission from the upper polariton mode (UP), lower polariton mode (LP), and direct emission from the uncoupled J-aggregate excitons, respectively. Fig. 2 (b) shows emission measured as a function of angle and wavelength. The emission peaks measured at each can be mapped to a classical dispersion relation of energy $E$ vs. momentum $k$ where $E=h c / \lambda$ where $h$ is Planck's constant, $c$ is the speed of light and $\beta$ is given by 1 . Emission from the fundamental TE and TM eigenmodes of the system can be identified in Fig. 2 (b). Both present anti-crossings between the optical mode and the exciton resonance, at around $\theta=61^{\circ}$ for TE and around $\theta=45^{\circ}$ for TM. This verifies that the measured leaky emission is from WGEP modes. We also perform angle resolved white light reflectivity measurements in a way similar to 22 . We simulate, using Fresnel reflection calculations, the four layer system comprising the BK7 glass prism, the silver film (40 nm thick), the PVA film (275 nm thick) doped with excitons, and the semi-infinite air section adjacent to the PVA film. To model the permittivity of the J-aggregate 

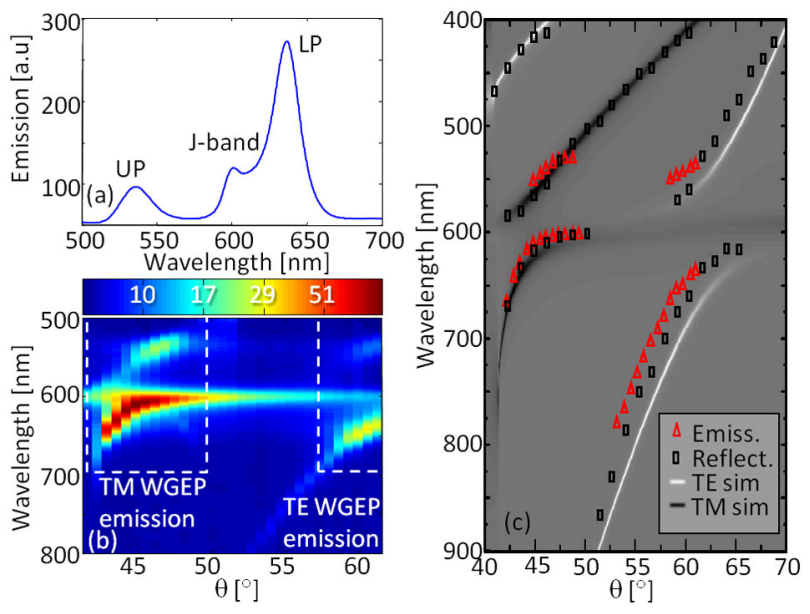

Figure 2: (color online). (a) Emission spectrum measured in the far field at $\theta=61.2^{\circ}$. (b) Angle resolved emission spectra (note that the color scale is logarithmic with arbitrary units). (c) Splitting of the dispersion curves of the system. Red triangles show measured emission peaks, black squares show measured reflectance dips and black and white curves show simulated angle resolved reflectance dips of p-polarized and s-polarized light respectively.

doped PVA film, we use a single Lorentzian oscillator model [22] with $\epsilon_{\infty}=2.4$ as the off-resonance permittivity of the film, $E_{0}=2074 \mathrm{meV}$ as the resonance energy and $\Delta E=51 \mathrm{meV}$ as the resonance width.

Fig. 2(c) shows the experimental measurements of emission peaks and reflectance dips imposed on the simulated reflectance. The red triangles correspond to emission peaks and the black squares correspond to reflectance dips. The white and black lines show the simulated reflectance dips of the TE and TM modes, respectively. The peaks in emission and dips in reflection measurements agree with the simulated results, verifying that the leaky emission from the system originates at strongly coupled WGEP modes.

In order to check the dependence of the coupling strength between the optical modes and the excitons, $g$, on the absorbance of the excitonic films, $\alpha$, we fabricate four samples $(\backsim 230 \mathrm{~nm}$ thick) with different concentrations of dye molecules at a ratio of $\backsim 1: 2: 4: 8$. White light reflectivity measurements are used to extract the dispersion relation of the eigenmodes of the different samples similar to Fig. 2(c) and the coupling strength is measured on an $E(\beta)$ space by $g=E_{U P}-E_{L P}$ where $E_{U P}$ and $E_{L P}$ are the resonant energies of the upper and lower WGEP respectively. The absorbance of the different samples is extracted by performing optical transmission measurements from the excitonic waveguide deposited directly on glass. The optical absorbance of the sample is $\alpha=-\ln \left(T / T_{0}\right)$ where $T_{0}$ and $T$ are the transmissions at the J-band through bare glass and excitonic film on glass, respectively. Fig. 3 plots the coupling strength as a func-

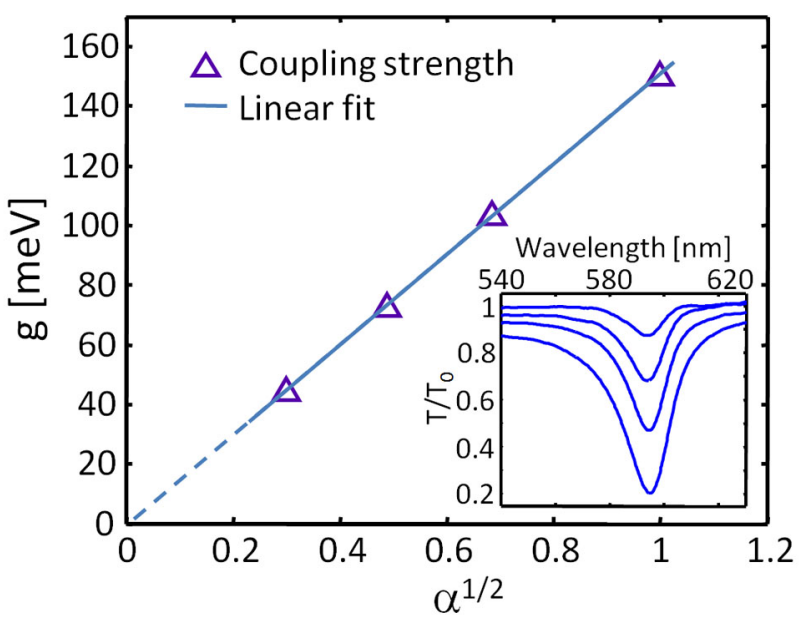

Figure 3: (color online). Coupling strength as function of the square root of the normalized absorbance in the doped PVA film. Inset shows transmittance measurements from four samples with different dye concentrations corresponding to the data point shown in the figure.

tion of the square root of the normalized absorbance. A linear relation occurs for the WGEPs, just as it does for cavity EPs. The inset shows the normalized transmission measurements from which the absorbance is found.

Changing the thickness of the exciton doped polymer waveguide presents a means for modifying the confinement and propagation properties of the fundamental modes of the waveguide, which in turn affects the effective indices, $n_{\text {eff }}$, of the modes. The momenta of the EPs, $\beta_{W G E P}=2 \pi n_{e f f} / \lambda$, can therefore be controlled. We fabricate samples with excitonic waveguide thicknesses $d$ of $275 \mathrm{~nm}, 230 \mathrm{~nm}$ and $195 \mathrm{~nm}$ by choosing the spin coating speed to be $2000 \mathrm{rpm}, 2500 \mathrm{rpm}$ and $3000 \mathrm{rpm}$, respectively. Fig. 4 (a)-(c) present angle resolved leaky emission measurements from these samples and Fig. 4 (d)-(f) show the corresponding simulated reflectance results. It is shown that increasing $d$ from 195 $\mathrm{nm}$ to $275 \mathrm{~nm}$ results in increasing the leaky emission angle by $\backsim 7^{\circ}$, which is corresponding to $\backsim 10 \%$ change in the momentum of the WGEP.

Non-resonant excitation of the system from the film side generates excitons which can relax into WGEPs. The spatial density of WGEP states in the slab waveguide geometry is radially symmetric. The excitons therefore relax into radially symmetric WGEPs (with $\beta_{T E}$ and $\beta_{T M}$ ) which originate at the pump spot. To create an image of the momentum space of the WGEP modes, we replace the prism of our set-up with a hemisphere. This enables the WGEPs to couple to free-space radiation modes, and the angle of emission to be readily converted to momentum using Eq. 1. Fig. 5(a) depicts the experimental setup. The sample is pumped with a $532 \mathrm{~nm} \mathrm{CW}$ laser and the waveguide modes are coupled out using the glass hemisphere optically coupled to the sample with in- 

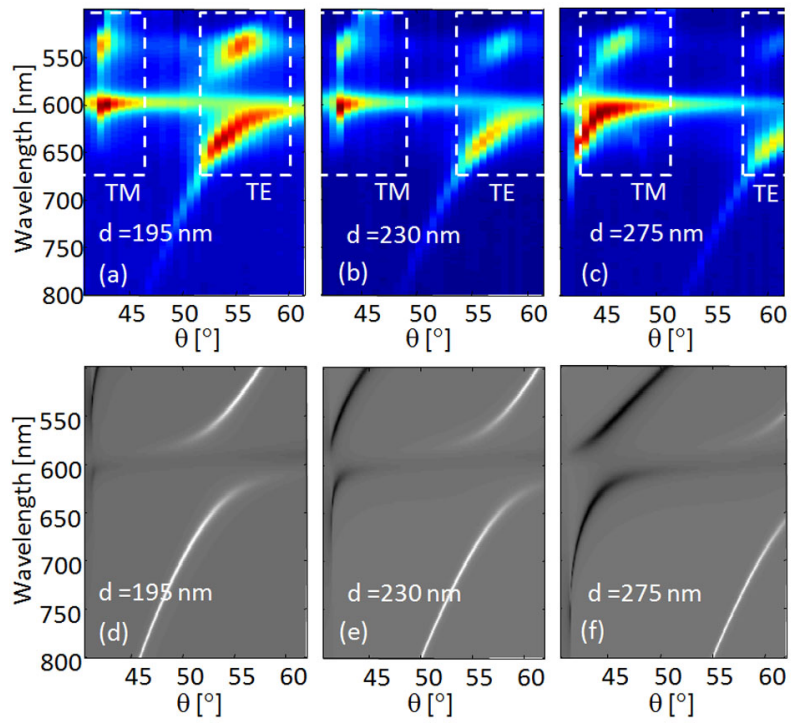

Figure 4: (color online). Angle resolved emission spectra from samples with (a) $195 \mathrm{~nm}$, (b) $230 \mathrm{~nm}$, and (c) $275 \mathrm{~nm}$ thicknesses and corresponding simulated reflectance results (d)-(f) where white lines correspond to TE modes and black lines to TM modes.

dex matching oil. The emitted light is filtered with a 550 $\mathrm{nm}$ long pass filter and incident upon a semitransparent screen. The screen is photographed by a camera from the other side, with the result shown as Fig 5(b). Two rings corresponding to two cones of light leaking out of the system can be seen. The inner cone corresponds to emission from the TM WGEP with $\beta_{T M}$ and the outer cone corresponds to the emission from the TE WGEP with $\beta_{T E}$. The cross polarized TM and TE waveguide modes are leaking to cross polarized light cones where the polarization of the TM WGEP cone is radial and the polarization of the TE WGEP is azimuthal. Fig. 5(c) shows the momentum space image obtained when a polarizing film is placed right after the hemisphere. The white arrow in the picture shows the direction of the polarization transmitted by the polarizing film. The crescent, rather than circular, shapes of the WGEP cones are consistent with the polarizations of the TM and TE WGEPs being radial and azimuthal, respectively.

In conclusion we study leaky emission from thin polymer films doped with J-aggregate molecules. We show that this emission originates at strongly coupled WGEP modes, and occurs at angles corresponding to the phase matching conditions between the strongly coupled light matter modes and leaky photons. The WGEP modes are studied by emission and reflectance measurements, which are seen to agree with reflectance simulations. Similar to cavity EPs, the coupling strength of WGEPs has a quadratic proportionality to the absorbance in the excitonic film. We show that the momentum of the waveguide modes can be modified by changing the thickness

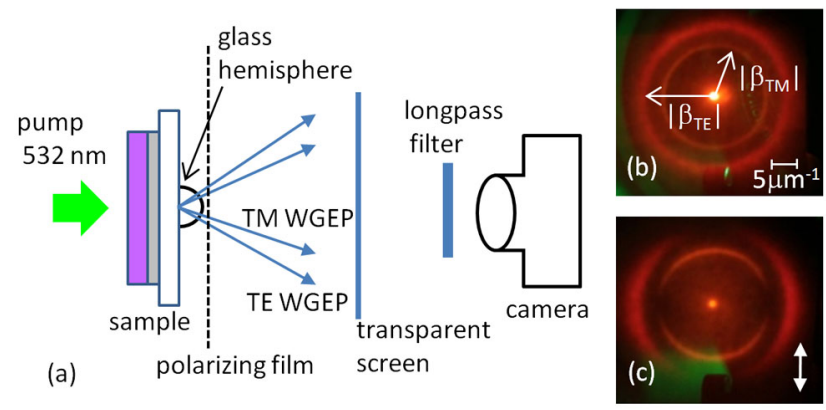

Figure 5: (color online). (a) Experimental setup used to measure the momentum space of the WGEP modes of the system. (b) Photographed momentum space of WGEPs. (c) Polarization dependence of the momentum space. The white arrow depicts the polarization of the transmitted light.

of the excitonic waveguide, in a similar way to regular optical waveguide modes. By capturing the $2 \mathrm{D}$ momentum space of the WGEP in the system, we demonstrate that non-resonant pumping of the excitons in the system relaxes to WGEPs with radial symmetry. These modes translate into cones of leaky free space emission with radial and azimuthal polarization states for the TM WGEP and TE WGEP respectively. We anticipate that the optical waveguide configuration with its differences and advantages over the optical cavity configuration could be used for new fundamental studies on EPs, e.g. by probing the modes in the near field through their evanescent tails, and will potentially lead to new applications based on light matter states, e.g. low-threshold all-optical switching of light in interconnects.

This work was funded by the Center for Excitonics, an Energy Frontier Research Center funded by the U.S. Department of Energy, Office of Science and Office of Basic Energy Sciences under Award Number DE-SC0001088.

* Electronic address: tale@seas.harvard.edu

† Electronic address: kcrozier@seas.harvard.edu

[1] B. E. A. Saleh and M. C. Teich, Fundamentals of Photonics, 2nd ed. (Wiley-Interscience, 2007).

[2] J. Zhang et al., Nature Rev. Mol. Cell Biol. 3, 906 (2002).

[3] C. Weisbuch et al., Phys. Rev. Lett. 69, 3314 (1992).

[4] G. Khitrova et al., Nature Phys. 2, 81 (2006).

[5] D. M. Whittaker et al., Phys. Rev. Lett. 77, 4792 (1996).

[6] F. Tassone and Y. Yamamoto, Phys. Rev. B 59, 10830 (1999).

[7] P.G. Savvidis et al., Phys. Rev. Lett. 84, 1547 (2000).

[8] H. Deng et al., Science 298, 199 (2002).

[9] J. Kasprzak et al., Nature 443, 409 (2006).

[10] R. Balili et al., Science 316, 1007 (2007).

[11] S. Kena-Cohen and S. R. Forrest, Nature Photon. 4, 371 (2010).

[12] S. Christopoulos et al., Phys. Rev. Lett. 98, 126405 (2007). 
[13] A. Amo et al., Nature Photon. 4, 361 (2010).

[14] D. Goldberg et al., Nature Photon. 3, 662 (2009).

[15] J. Bellessa et al., Phys. Rev. Lett. 93, 036404 (2004).

[16] T. K. Hakala et al., Phys. Rev. Lett. 103, 053602 (2009).

[17] N. T. Fofang et al., Nano Lett. 8, 3481 (2008).

[18] T. Katsuyama and K. Ogawa, J. Appl. Phys. 75, 76077625 (1994).

[19] L. K. van Vugt et al., Phys. Rev. Lett. 97, 147401 (2006).
[20] K. Takazawa et al., Phys. Rev. Lett. 105, 067401 (2010).

[21] M. Liscidini et al., Appl. Phys. Lett. 98, 121118 (2011).

[22] T. Ellenbogen, P. Steinvurzel and K. B. Crozier, Appl. Phys. Lett. 98, 261103 (2011)

[23] M. van Burgel, D. A. Wiersma and K. Duppen, J. Chem. Phys. 102, 20 (1994).

[24] D. Cristea et al., Mat. Sci. Eng. C 26, 1049 (2006). 